[0212-7199 (2004) 21: 11; pp 551-553] ANALES DE MEDICINA INTERNA Copyright (C) 2004 ARAN EDICIONES, S.L.

AN. MED. INTERna (Madrid) Vol. 21, N. ${ }^{\circ} 11$, pp. 551-553, 2004

\section{Anticoagulación con bemiparina tras hemorragia intracerebral como complicación de endocarditis bacteriana sobre válvula protésica metálica}

\author{
A. MUÑOZ MORENTE, M. A. BARÓN RAMOS, S. MATEOS FERNÁNDEZ1, \\ S. LÓPEZ PALMERO, J. VILLAR JIMÉNEZ, J. M. REGUERA IGLESIAS
}

Servicio de Medicina Interna. 'Servicio de Urgencias y Cuidados Críticos.

Hospital Regional Carlos Haya. Málaga

ANTICOAGULATION WITH BEMIPARINA AFTER INTRACEREBRAL TIS ON METALLIC PROSTHETIC VALVE

\section{RESUMEN}

Presentamos el caso de un paciente con una hemorragia intracerebral como complicación de una endocarditis infecciosa (EI) producida por Neisseria sicca sobre una válvula mitral metálica. El paciente estaba previamente anticoagulado con Acenocumarol como profilaxis de trombosis de la válvula protésica. Fue diagnosticado de EI y posteriormente presentó síntomas neurológicos como consecuencia de varios focos hemorrágicos intracerebrales. Se decidió continuar la anticoagulación con heparina sódica inicialmente y posteriormente con Bemiparina, no observándose nuevas complicaciones hemorrágicas ni trombosis de la válvula a los tres meses del evento. No hemos encontrado en la literatura ningún caso donde la heparina de bajo peso molecular (HBPM) haya sido utilizada como método de anticoagulación en estos casos más allá de dos semanas.

PALABRAS CLAVE: Endocarditis infecciosa. Neisseria sicca. Hemorragia intracerebral. Heparina bajo peso molecular.

\begin{abstract}
We present the case of a patient with an intracerebral hemorrhage as complication of an infectious endocarditis (EI) produced by Neisseria sicca on a prosthetic mitral valve. The patient was anticoagulated previously with Acenocumarol as prophylaxis of thrombosis of the prosthetic valve. He was diagnosed as having IE and later he presented neurological symptoms as consequence of several intracerebral hemorrhagic foci. We decided to continue the anticoagulation with sodium heparine followed of Bemiparina and no new hemorrhagic complications nor thrombosis of the valve were observed after three months of the event. We have not found in the literature any case where low molecular weight heparine has been used as method of anticoagulation in these cases beyond two weeks.
\end{abstract}

KEY WORDS: Infectious endocarditis. Neisseria sicca. Intracerebral hemorrhage. Low molecular weight heparine.

Muñoz Morente A, Barón Ramos MA, Mateos Fernández, S, López Palmero S, Villar Jiménez J, Reguera Iglesias JM. Anticoagulación con bemiparina tras hemorragia intracerebral como complicación de endocarditis bacteriana sobre válvula protésica metálica. An Med Interna (Madrid) 2004: 21:551-553.

\section{INTRODUCCIÓN}

Las complicaciones neurológicas en las EI izquierdas tienen una incidencia que puede alcanzar hasta un $40 \%$ (1). Las hemorragias intracerebrales, aunque poco frecuentes $(<5 \%)$, presentan una elevada mortalidad $(60-80 \%)$ (2) y suelen deberse a la transformación hemorrágica de un infarto previo, sobre todo en aquellos pacientes que están previamente anticoagulados (3). El manejo de los pacientes con prótesis valvulares metálicas infectadas y hemorragia cerebral es complicado, ya que, la necesidad de mantener la anticoagulación para evitar la trombosis valvular pone en riesgo al paciente para nuevos episodios hemorrágicos cerebrales. Sin embargo, no existe un consenso en la literatura sobre cuál debe ser el manejo de estas situaciones. Exponemos el caso de un paciente con EI y hemorragia intracerebral en el que optamos por sustituir el Acenocumarol por heparina sódica y posteriormente por Bemiparina, una heparina de bajo peso molecular que puede ser administrada una vez al día, y que mantuvimos durante tres meses sin que evidenciáramos nuevos sangrados cerebrales ni trombosis valvular a través de ecocardiografía. No hemos encontrado ningún caso de similares características donde la HBPM haya sido usada por un período mayor a dos semanas.

Trabajo aceptado: 6 de mayo de 2004

Correspondencia: Ángel Muñoz Morente. C/ Hotel Pontinental, 16. Residencial El Limonar. $3^{\circ}$ A. Playamar. 29620 Torremolinos (Málaga). e-mail: amcubo@telefonica.net 


\section{CASO APORTADO}

Paciente varón de 60 años de edad, con prótesis mitral metálica tipo Björk-Shilley y anuloplastia tricuspídea 20 años atrás por valvulopatía reumática, fibrilación auricular crónica (FAC), HTA, insuficiencia cardiaca congestiva (ICC) con un Grado Funcional NYHA I/IV. Recibía tratamiento con acenocumarol. Ingresa en nuestro servicio por cuadro de fiebre, escalofríos, y empeoramiento de su disnea habitual, apareciendo ortopnea y edemas en MMII. La exploración clínica era compatible con una ICC (venas yugulares ingurgitadas, pulso arritmico a $100 \mathrm{lpm}$, ritmo de galope, crepitantes basales bilaterales pulmonares, hepatomegalia de 2 traveses con reflejo hepatoyugular positivo y edemas pretibiales con fóvea en los MMII). En la analítica presentaba una hemoglobina de 12,4 $\mathrm{g} / \mathrm{L}$, leucocitos $17.810 / \mathrm{ml}$, con un $87 \%$ de neutrófilos, estando el resto de los parámetros habituales dentro de la normalidad. La radiografía de tórax mostraba signos de ICC. Se realizaron hemocultivos seriados en los que se aisló de forma persistente Neisseria Sicca, por lo que se inició tratamiento con Penicilina G sódica intravenosa 24 millones de unidades en perfusión contínua y Gentamicina $80 \mathrm{mg} \mathrm{I}$.V. cada 8 horas (2 primeras semanas), quedando el paciente afebril al tercer día de comenzar el tratamiento. En el transcurso del ingreso aparecieron unas lesiones rojizas y dolorosas en los pulpejos de los dedos de ambas manos compatibles con fenómenos vasculíticos, realizándose, por tanto, el diagnóstico de Endocarditis Infecciosa (EI) según los criterios clínicos de Durack (4). La Ecocardiografia Transtorácica (ETT) y Transesofágica (ETE) demostró una prótesis mitral normofuncionante y ausencia de vegetaciones. Al $9^{\circ}$ día de tratamiento el paciente presentó un episodio brusco de pérdida de fuerza en hemicuerpo izquierdo, visualizándose en TAC craneal 3 imagénes hiperdensas a nivel temporalparietal y occipital de ambos hemisferios compatibles con focos hemorrágicos. En ese momento en I.N.R. era de 2,45, a pesar de lo cual, la anticoagulación fue suspendida. Cuatro días después, y la tras estabilización del paciente, se decidió reiniciar anticoagulación con heparina sódica iv completándose 6 semanas de tratamiento antibiótico y heparina. En el TAC cerebral de control tras el tratamiento, persistía el hematoma occipitoparietal, habiendo desaparecido los otros focos hemorrágicos. Ante éstos hallazgos y la posibilidad de resangrado, el paciente fue dado de alta con bemiparina 10.000 U.I. subcutánea una vez al día, durante 3 meses, tras los cuales se realizó nuevo estudio ecocardiográfico y TAC craneal que demostraron la normalidad de la válvula mitral y la práctica resolución del hematoma cerebral, por lo que nuevamente se inició anticoagulación oral con acenocumarol, encontrándose a los tres meses de la misma, asintomático.

\section{DISCUSIÓN}

La infección de las prótesis valvulares metálicas continúa siendo una seria complicación aunque con una relativa baja incidencia $(0,1-2,3 \%$ paciente-año), si bien, últimamente se está incrementando (5). Al mismo tiempo, las hemorragias cerebrales son de las complicaciones más peligrosas de la EI debido a su alta mortalidad. Por otro lado, la incidencia de trombosis de la válvula metálica en pacientes no anticoagulados ni antiagregados es de $1,8 \%$ paciente-año, siendo la incidencia de embolismo, accidente cerebrovascular o isquemia periférica del $4 \%$, que se reduce al $2,2 \%$ con antiagregación y al $1 \%$ con anticoagulación oral (6), por lo que la necesidad de mantener anticoagulación oral en pacientes con prótesis valvular metálica parece evidente y está ampliamente aceptado. Pero, la hemorragia intracerebral puede ser una complicación de la anticoagulación oral en aproximadamente un $1 \%$ pacientes-año, es decir, unas 7-10 veces más fre- cuente que en pacientes no anticoagulados, con una mortalidad que varía entre el 20-80\% (7). En el caso descrito nos enfrentamos a un paciente con una EI sobre una válvula protésica metálica, con alto riesgo tromboembólico, al presentar hipertensión, FAC y prótesis metálica mitral tipo Bjork-Shiley (8), por lo cual, inicialmente se mantuvo la anticoagulación de acuerdo con la opinión de diversos autores $(9,10)$, aunque no haya consenso unánime al respecto, máxime cuando se trata de gérmenes muy virulentos (11). Pero tras confirmarse la hemorragía cerebral, la anticoagulación fue suspendida para evitar la progresión de los focos hemorrágicos. Sin embargo, dado el riesgo de trombosis valvular y/o tromboembolismo, la anticoagulación debía ser reintroducida. El momento de reiniciar la anticoagulación fue controvertido, ya que existen escasas evidencias en la literatura de cuál es el mejor momento. Además, casi todos los estudios publicados a este respecto están realizados en pacientes sin EI. Phan y cols. (12) en un estudio de 141 pacientes con anticoagulación oral y hemorragia intracerebral -de los cuales 52 tenían prótesis metálica- concluyen que la suspensión de la anticoagulación durante 2 semanas es segura, estimando el riesgo tromboembólico en un $3 \%$ en los primeros 30 días de cese de anticoagulación. Sin embargo, en este estudio, los infartos hemorrágicos fueron excluidos y no hubo seguimiento posterior tras los primeros 30 días. Butller y Tait (13) en 16 pacientes con prótesis metálica, encuentran resultados similares, si bien durante su estudio, 10 de los pacientes recibieron heparina durante una media de 3 días tras el sangrado y solo 2 alcanzaron dosis terapéuticas (TPTA 1,5-2,5). Wijdicks y cols. (14) en un estudio sobre 39 pacientes analizan por separado a 9 pacientes con alto riesgo tromboembólico a los que suspende anticoagulación por al menos una semana, sin ocurrir ningún fenómeno embolígeno. En otro estudio, sobre solo 4 pacientes, encuentran que reiniciar la anticoagulación con heparina intravenosa en las primeras 24-36 horas podría ser una alternativa segura (15). En nuestro caso, se reinició la anticoagulación cuando el paciente se encontraba estable y sin signos de empeoramiento del sangrado intracerebral.

Ante la persistencia del hematoma en TAC craneal de control una vez finalizado el tratamiento antibiótico del paciente, se decidió continuar la anticoagulación con bemiparina. Son numerosos los artículos que hacen referencia a la seguridad del uso de HBPM por un corto intervalo de tiempo durante el cual se requiere suspender la anticoagulación oral previo a la realización de técnicas invasivas, cirugía, etc. (16-18), equiparándose incluso a heparina no fraccionada tras cirugía de recambio valvular. Sin embargo, son escasas las referencias en la literatura sobre el manejo con HBPM durante un tiempo prolongado de estos pacientes. Lev-Ran (19) describe un paciente con prótesis aórtica metálica tratado con enoxaparina tras una hemorragia cerebral, presentando a los 9 meses del inicio de este tratamiento obstrucción de la válvula, si bien, el paciente estaba siendo tratado a dosis infraterapéuticas (40 mg dos veces al día).

En conclusión, el manejo de la EI sobre válvula protésica con hemorragia cerebral es complicado y requiere una gran experiencia. No existe un consenso sobre la forma de actuar en estos casos, de modo que, se necesita la realización de estudios prospectivos que ayuden a determinar cuál es el manejo más correcto en este tipo de pacientes, la forma de anticoagulación más segura y el tiempo de seguimiento. 


\section{Bibliografía}

1. Pruitt AA, Rubin RH, Karchmer AW, Duncan GW. Neurologic complications of bacterial endocarditis. Medicine 1978; 57: 329-43.

2. Hart RG, Kagan-Hallet K and Joerns SE. Mechanisms of intracranial hemorrhage in infective endocarditis. Stroke 1987;18: 1048-56.

3. Masuda J, Yutani Ch, Waki R, Ogata J, Kuriyama Y, Yamaguchi T. Histopathological analysis of the mechanisms of intracranial hemorrhage complicating infective endocarditis. Stroke 1992; 23: 843-50.

4. Durack et al. New criteria for diagnosis of infective endocaditis: utilización of specific echocardiographic findings. Am J Med 1994; 96 : 200-9.

5. Piper C, Körfer R, Horstkotte D. Prosthetic valve endocarditis. Heart 2001; 85: 590-3.

6. Cannegieter SC, Rosendaal FR, Briët E. Thromboembolic and bleeding complications in patients with mechanical heart valve prostheses. Circulation 1994; 89: 635-41.

7. Gohlke-Bärwolf C. Anticoagulation in valvar heart disease: new aspects and management during non-cardiac surgery. Heart 2000; 84: 567-72.

8. Hart RG, Boop BS, Anderson DC. Oral anticoagulants and intracranial hemorrhage: facts and hypotheses. Stroke 1995; 26: 1471-7.

9. Wilson WR, Geraci JE, Danielson GK, et al. Anticoagulant therapy and central nervous system complications in patients with prosthetic valve endocarditis. Circulation 1978; 57: 1004-7.

10. Vongpatanasin W, Hillis LD, Lange RA. Prosthetic heart valves. NEJM 1996; 335: 407-16.

11. Tornos P, Almirante B, Mirabet S, Permanyer G, Pahissa Albert, Soler-Soler J. Infective endocarditis due to Staphylococcus aureus: deleterious effect of anticoagulant therapy. Arch Intern Med 1999; 159: 473-5.

12. Phan TG, Koh M, Wijdicks EF. Safety of discontinuation of anticoagulation in patients intracranial hemorrhage at high thromboembolic risk. Arch Neurol 2000; 57: 1710-3.

13. Butler AC, Tait RC. Restarting anticoagulation in prosthetic heart valve patients after intracranial haemorrhage: a 2-year follow-up. $\mathrm{Br} \mathrm{J}$ Haematol 1998; 103: 1064-6.

14. Wijdicks EF, Schievink WI, Brown RD, Mullany CJ. The dilemma of discontinuation of anticoagulation therapy for patients with intracranial hemorrhage and mechanical heart valves. Neurosurgery 1998;42: 769-73.

15. Leker RR, Abramsky O. Early anticoagulation in patients with prosthetic heart valves and intracerebral hematoma. Neurology 1998; 50: 1489-91.

16. Shapira Y, Sagie A, Battler A. Low-molecular-weight heparin for the treatment of patients with mechanical heart valves. Clin Cardiol 2002; 25: 323-7.

17. Ferreira I, Dos L, Tornos P, Nicolau I, Permanyer-Miralda G, SolerSoler J. Experience with enoxaparina in patients with mechanical heart valves who must withhold acenocumarol. Heart 2003; 89: 527 30.

18. Montalescot $\mathrm{G}$, et al. Low molecular weight heparine after mechanical heart valve replacement. Circulation 2000; 101: 1083-6.

19. Lev-Ran O, Kramer A, Gurevitch J, Shapira I, Mohr R. Low-molecular-weight heparin for prosthetic heart valves: treatment failure. Ann Thorac Surg 2000; 69: 264-6. 